\title{
In Vitro Analysis of Immersed Human Tissues by Raman Microspectroscopy
}

\author{
Franck Bonnier \\ Technological University Dublin, Franck.Bonnier@tudublin.ie
}

A. Mehmood

Technological University Dublin

Peter Knief

Technological University Dublin, peter.knief@tudublin.ie

See next page for additional authors

Follow this and additional works at: https://arrow.tudublin.ie/radart

Part of the Atomic, Molecular and Optical Physics Commons

\section{Recommended Citation \\ Bonnier, F. et al. (2011) In vitro Analysis of ilmmersed Human Tissues by Raman Microspectroscopy. Journal of Raman Spectroscopy, 42: pp.888-896. doi:10.1002/jrs.2825}

This Article is brought to you for free and open access by the Radiation and Environmental Science Centre at ARROW@TU Dublin. It has been accepted for inclusion in Articles by an authorized administrator of ARROW@TU Dublin. For more information, please contact arrow.admin@tudublin.ie, aisling.coyne@tudublin.ie, gerard.connolly@tudublin.ie.

Funder: The National Biophotonics and Imaging Platform (NBIP) Ireland funded under the Higher Education Authority PRTLI (Programme for Research in Third Level Institutions) Cycle 4, co-funded by the Irish Government and the European Union.

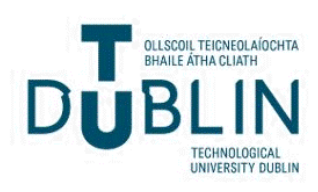




\section{Authors}

Franck Bonnier, A. Mehmood, Peter Knief, Aidan Meade, Helen Lambkin, Kathleen Flynn, V. McDonagh, C. Healy, T. C. Lee, Fiona Lyng, and Hugh Byrne 


\title{
In vitro analysis of immersed human tissues by Raman microspectroscopy
}

\author{
F. Bonnier ${ }^{1}$, A.Mehmood ${ }^{1}$, P. Knief ${ }^{1}$, A. Meade ${ }^{5}$, W. Hornebeck ${ }^{2}$, H. Lambkin ${ }^{3}$, K. \\ Flynn $^{3}$, V. McDonagh ${ }^{4}$, C. Healy ${ }^{4}$,T.C. Lee ${ }^{4}$, F.M. Lyng ${ }^{1}$, H.J. Byrne ${ }^{1}$ \\ ${ }^{1}$ Focas Research Institute, Dublin Institute of Technology, Kevin Street, Dublin 8, \\ Ireland \\ ${ }^{2}$ URCA- Faculty of Medicine - CNRS UMR 6237 MeDYC, Université de Reims- \\ Champagne Ardenne, 51 rue Cognacq-Jay, 51096 Reims Cedex, France. \\ ${ }^{3}$ School of Biological Sciences, Dublin Institute of Technology, Kevin Street, Dublin \\ 8, Ireland. \\ ${ }^{4}$ Department of Anatomy, Royal College of Surgeons in Ireland, 123 St. Stephen’s \\ Green, Dublin 2, Ireland. \\ ${ }^{5}$ School of Physics, Dublin Institute of Technology, Kevin Street, Dublin 8, Ireland
}

\begin{abstract}
:
Raman microspectroscopy is a powerful tool for the analysis of tissue sections providing a molecular map of the investigated samples. Nevertheless, data pre processing, and particularly the removal of broad background to the spectra remains problematic. Indeed, the physical origin of the background has not been satisfactorily determined. Using $785 \mathrm{~nm}$ as source in a confocal geometry, it is demonstrated, that for the example of the protein kappa-elastin, the background and resulting quality of the recorded spectrum is dependent on the morphology of the sample. Whereas a fine powder yields a dominant broad background, compressed pellets and solution cast thin films produce respectively improved quality spectra with significantly reduced spectral background. As the chemical composition of the samples is identical, the background is ascribed to stray light due to diffuse scattering rather than an intrinsic photoluminescence.

Recorded spectra from tissue sample exhibit a large and spatially variable background, resulting in poorly defined spectral features. A significant reduction of the background signal and improvement of the spectral quality is achieved by immersion in water, and measurement with an immersion objective. The significant improvement in signal to background is attributed to a reduction of the diffuse scattering due to a change in the effective morphology as a result of an improved index matching between the water/ tissue interface compared to the air/tissue interface.

Compared to sections measured in air, the background is reduced to that of the water, and preprocessing is reduced to the subtraction of the substrate and water signal, and correction for the instrument response, all of which are highly reproducible. Data preprocessing is thus greatly simplified and the results significantly more reliable.
\end{abstract}

Keywords: Raman Spectroscopy; scattering, tissue analysis; dewaxing; refractive index

\footnotetext{
* Corresponding author. Tel.: +353 1 4027917; Fax: +353 1 4027904;

E-mail address: fbonnier@dit.ie (F. Bonnier)
} 


\section{Introduction}

Vibrational spectroscopic techniques are widely used for the investigation of biological samples from the cellular and subcellular level to tissue sections ${ }^{[1-4]}$. Infrared and Raman spectroscopy are both powerful approaches giving access to the molecular composition of the samples studied. The main advantage of Raman spectroscopy is the possibility to record spectra from live cells using an immersion objective ${ }^{[5,6]}$. Water having a very strong absorption in the infrared, the samples usually have to be dried before analysis using this complementary technique ${ }^{[7]}$.

At present, the main challenge is the development of standardised methods for data analysis ${ }^{[8]}$. Different approaches such as Principal Component Analysis (PCA) or Clustering K-means are commonly used for statistical analysis of large amounts of data, allowing a discrimination of different samples or regions of a sample according to differences in their biochemical content and identification of the spectral features containing the most variability ${ }^{[9-13]}$. Although these methods are used routinely and are quite well developed, the question of the pre-processing of the data before analysis is still of concern.

In Fourier Transform Infrared microscopy, the physical origins of the broad undulating background ${ }^{[14]}$ and the so-called dispersion artefact in transflection ${ }^{[15]}$ and transmission ${ }^{[16]}$ mode have recently been elucidated and the Extended Multiplicative Scattering Correction has been further evolved to correct for both ${ }^{[17,18]}$. In Raman spectroscopy, the most commonly reported artefact is the presence of a broad background which can often swamp the sample signal ${ }^{[19,20]}$. Although different 
algorithms can be applied to subtract this background from the spectra ${ }^{[21]}$, to date it has not been satisfactorily modelled in terms of physical origin and thus it is difficult to establish when it has been correctly removed.

Raman spectroscopy is widely used for diagnostic purposes as it has the potential advantage of being adaptable to in-vivo measurements ${ }^{[22-24]}$. Thus many studies are focused on the study of thin tissue sections in order to visualise and highlight modifications in the molecular composition of the samples which could be used as markers for the discrimination between tissues in different diseased states ${ }^{[25,26]}$. Embedding in paraffin wax is commonly undertaken to conserve the samples before histological analysis ${ }^{[27,28]}$ and archived tissue banks are a significant resource in terms of demonstrating and optimising the techniques as well as establishing a relationship to disease prognosis. Although the paraffin has an intense Raman signal overlapping with the molecular vibrations from the sample, after chemical dewaxing the thin sections can be used for Raman spectroscopy ${ }^{[29]}$. However, the Raman spectra recorded from such samples commonly exhibit a profile in which the spectral features of the tissue are swamped by a substantial background. Although digital dewaxing has been proposed to avoid the use of chemicals for the removal of the paraffin ${ }^{[30]}$, the presence of wax in the sections studied significant reduces the contrast in optical images and restricts the identification of the different structures present in the sections analysed. Thus, chemical dewaxing remains the best option for tissue imaging using Raman microspectroscopy. Experimentally, using longer wavelength sources in the near infrared reduces the background and also minimises the possibility of photochemical or photothermal damage during the measurements ${ }^{[6]}$. In confocal operation, the contribution of the substrate can also be reduced ${ }^{[31]}$. However, as shown in Figure 1A for the example of a de-waxed tissue section 
recorded using $785 \mathrm{~nm}$ as source in confocal mode, a substantial background remains. For comparison, this effect is not observed in the spectra of collagen type I recording using identical settings (Fig. 1B).

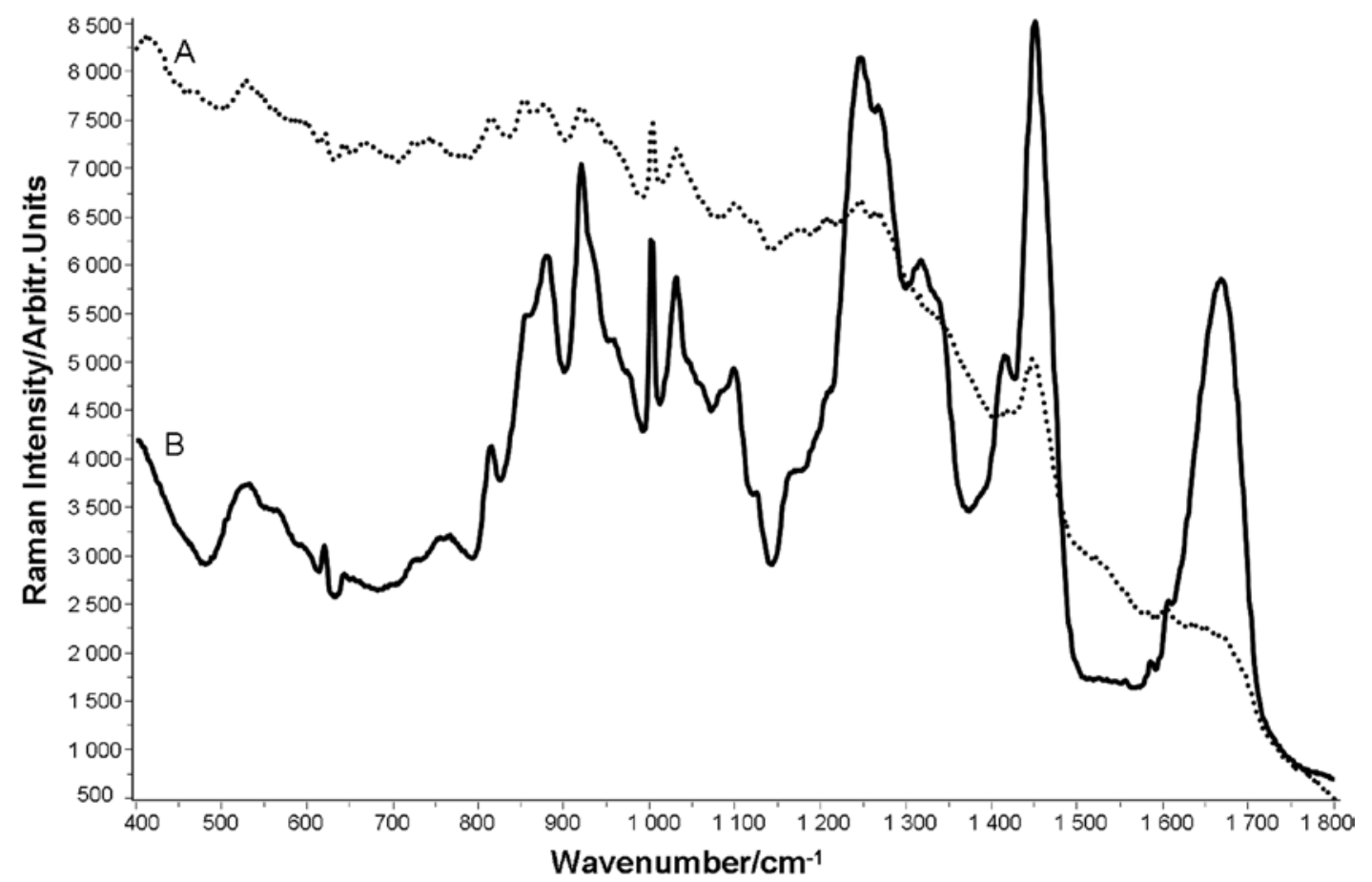

Fig.1 : Raman mean recorded spectrum on a human skin from the thigh section (10 $\mu$ m thick) after dewaxing using xylene (A) compared to a mean spectrum of type I collagen from rat tail (B). No correction has been applied on the spectra.

For diagnostic applications, ideally, spectral responses should be strong, such that cells and tissue can be rapidly screened and have a strong signal to background ratio such that subtle changes in signal can be observed and analysed. Using model protein samples, this work demonstrates that the background present in the spectra is related to scattering of the light due to the morphology rather than chemical content of the samples studied. In human thoracic aortas and human skin samples, performing the measurement on immersed samples results in a dramatic reduction of the background, facilitating spectral pre-processing and a more accurate interpretation of the acquired 
spectra. The observation is interpreted in terms of a reduction of stray light from scattered due to the reduced refractive index difference at the tissue/water interface in comparison to the tissue/air interface.

\section{Materials and Methods}

\subsection{Collagen and elastin samples}

Collagen spectra were recorded from a type I collagen sample derived from rat tail (GIBCO). A few microliters of an aqueous solution were dropped on a $\mathrm{CaF}_{2}$ window and dried before recording the Raman spectra.

Insoluble elastin was prepared from bovine ligamentum nuchae by hot alkali treatment and its purity was assessed by amino acid analysis and lack of hexoses and hexosamines in the preparation ${ }^{[32]}$. Soluble kappa-elastin peptides were obtained from purified insoluble elastin by organo-alkaline hydrolysis. Peptides with an average molecular weight of $75 \mathrm{kDa}$ were isolated by gel permeation on Sephadex G100. The powder obtained was used for recording Raman spectra before and after compaction into a disc. The kappa-elastin can be dissolved in acetic acid, and a few microliters of the solution were dropped on a $\mathrm{CaF}_{2}$ window and dried before measurement of Raman spectra.

\subsection{Tissue section preparation}

All tissues were obtained from the Anatomical Gift Programme from the Royal College of Surgeons in Ireland. All specimens were automatically wax embedded as follows: (a) vacuum fixed in 10\% buffered formal saline histograde, $\mathrm{pH}$ 6.8-7.2 (J.T.Baker; Deventer, The Netherlands) heated to $35^{\circ} \mathrm{C}$, (b) vacuum dehydration in 
industrial methylated spirits IMS T100 (Lennox; Dublin, Ireland) heated to $35^{\circ} \mathrm{C}$, (c) vacuum clearing in xylene (Serosep; Limerick, Ireland) heated to $35^{\circ} \mathrm{C}$, and (d) vacuum impregnation with Tissue-Tek III Embedding Wax with polymer added (Sakura Zoeterwoude, The Netherlands) and heated to $59^{\circ} \mathrm{C}$.

Dewaxing using Xylene: After embedding, the samples were sliced into $10 \mu \mathrm{m}$ sections using a microtome, and placed on $\mathrm{CaF}_{2}$ slides before being dried in an oven at $55^{\circ} \mathrm{C}$. The protocol for the dewaxing consists of immersion of the samples in a series of baths starting with two baths of xylene (BDH; Dorset, UK) for 5 min and 4 min, respectively, then two baths of Ethanol Absolut (Merck; Dorset, UK) for 3 min and 2 min, respectively, and finally a bath of Industrial Methylated Spirits 95\% (Lennox) for $1 \mathrm{~min}$.

Dewaxing using Hexane: the same procedure was used for dewaxing using Hexane (BDH), whereby xylene was substituted with hexane and the slides were kept immersed in this reagent for $18 \mathrm{hrs}^{[29]}$.

\subsection{Raman spectroscopic measurements}

A Horiba Jobin-Yvon LabRAM HR800 spectrometer with an external 300 mW diode laser operating at $785 \mathrm{~nm}$ as source was used throughout this work. For the measurements, either a x100 objective (MPlanN, Olympus) or a x100 immersion objective (LUMPlanF1, Olympus) were employed, each providing a spatial resolution of $\sim 1 \mu \mathrm{m}$ at the sample. The confocal hole was set at $100 \mu \mathrm{m}$ for all measurements, the specified setting for confocal operation. The system was spectrally calibrated to the $520.7 \mathrm{~cm}^{-1}$ spectral line of silicon and the intensity response function was corrected 
using the Standard Reference Material (SRM) No. 2243 of the National Institute of Standards, Boulder, Colorado, USA (NIST SRM 2243, 2242, 2241) ${ }^{[33]}$.

The LabRAM system is a confocal spectrometer that contains two interchangeable gratings (300 and 900 lines/mm respectively). In the following experiments the 300 lines/mm grating was used, providing a spectral dispersion of approximately $1.5 \mathrm{~cm}^{-1}$ per pixel. The backscattered Raman signal was integrated for 30 seconds over the spectral range from 400 to $1800 \mathrm{~cm}^{-1}$ and accumulated 2 times to improve the signal to noise ratio. The detector used was a 16-bit dynamic range Peltier cooled CCD detector. Images of the sample were acquired using a video camera within the system.

The samples were first recorded using the MPlanN Olympus objective in a dry state and then the same sections were immersed in distilled water before being recorded with the LUMPlanF1 Olympus objective. All the spectra from the skin samples were recorded from the dermis, particularly rich in collagen, whereas the spectra from the aorta were obtained from the media, mostly composed of elastin fibers ${ }^{[34,35]}$. These locations were selected in order to reduce in as much as possible the variability due to differences in the biochemical composition between the different samples. The observations made throughout this study are based on measurements realized on 5 different patients for both the ascending aortic walls and the human skin samples. Concerning the human skin samples, for each patient, 2 different locations have been tested: hands and thighs. Mapping of large areas verified the repeatability of the observations made. For clarity, only typical examples have been presented in this paper.

\section{Results and discussion}

1. Effect of the sample preparation on the Raman spectra: Kappa elastin as a model 
To visualise the effect of the sample preparation on the signal recorded, Raman spectra were recorded on three different kappa-elastin samples. The first spectrum was recorded on the powder dispersed on a $\mathrm{CaF}_{2}$ slide. The spectrum is displayed in figure 2A. A significant background is present making it impossible to visualise and identify specific peaks ${ }^{[36,37]}$. The amide I band, usually observed around $1650 \mathrm{~cm}^{-1}$, is totally absent, and the peaks at $1004 \mathrm{~cm}^{-1}$ and $1450 \mathrm{~cm}^{-1}$, respectively related to symmetric ring breathing of the phenylalanine and the $\mathrm{CH}$ deformation, can hardly be seen. The corresponding laser spot, visualised using the live video camera, is presented and it is noticeable that it was not possible to achieve a sharp focus of the spot on the powder. As the fine particles of the Kappa-elastin powder are significantly smaller than the laser spot, both the diffusive nature of the focal point and the spectrum recorded were seen to be independent of position on the sample. Consequently, the powder was then pressed to obtain a pellet using a hydraulic press before recording Raman spectra (fig. 2B). Although the spectrum still contains a significant background, the spectral features are more defined and it is now possible to identify different molecular vibrations present in the Kappa-elastin ${ }^{[36,37]}$. It should be noted that although the spectra are recorded under identical conditions, they are not quantitatively comparable in terms of absolute background, as the surface coverage on the substrate differs significantly. However, a dramatic improvement in signal to background and signal to noise is apparent. The amide I band is clearly visible around $1650 \mathrm{~cm}^{-1}$, and the peaks at $1004 \mathrm{~cm}^{-1}$ and $1450 \mathrm{~cm}^{-1}$ are better defined. More detailed protein features are also observable in the spectral range $800-1000 \mathrm{~cm}^{-1}$ and $1100-$ $1350 \mathrm{~cm}^{-1}$. The laser spot was found to be smaller and better defined on the pellet. Kappa-elastin being soluble in acetic acid, a suspension was prepared, dropped onto a 
$\mathrm{CaF}_{2}$ slide and dried before measurements. The first observation was the improvement in the laser spot size and it was now easier to define the focal position on the sample (fig.2C). The spectrum obtained clearly contains more details about the molecular composition of the kappa-elastin and, with a significantly reduced background, the relative amplitudes of the peaks have been greatly increased. The visibility of the peaks located at $621 \mathrm{~cm}^{-1}$ (C-C twisting of the phenylalanine), $889 \mathrm{~cm}^{-1}\left(\mathrm{CH}_{2}\right), 932$ $\mathrm{cm}^{-1}$ (C-C backbone) and $1341 \mathrm{~cm}^{-1}(\mathrm{CH})$ is a clear illustration of the detrimental effect of a strong background present in the spectra. These peaks were not visible in the spectra obtained from powder and are clearly defined in the recorded spectra after preparation of the sample from a suspension.

The spectra in figure 2A,B and C are recorded from chemically identical materials. Although the broad background present in Raman spectra is frequently interpreted as fluorescence $^{[19,20,38]}$, this is clearly not the case here. Kappa elastin is transparent at $785 \mathrm{~nm}$, and the signal to background ratio is dependent on sample morphology. Furthermore, it is noticeable that the signal to noise ratio increases, indicating that the morphology impact not only on the background levels but also the signal itself. 

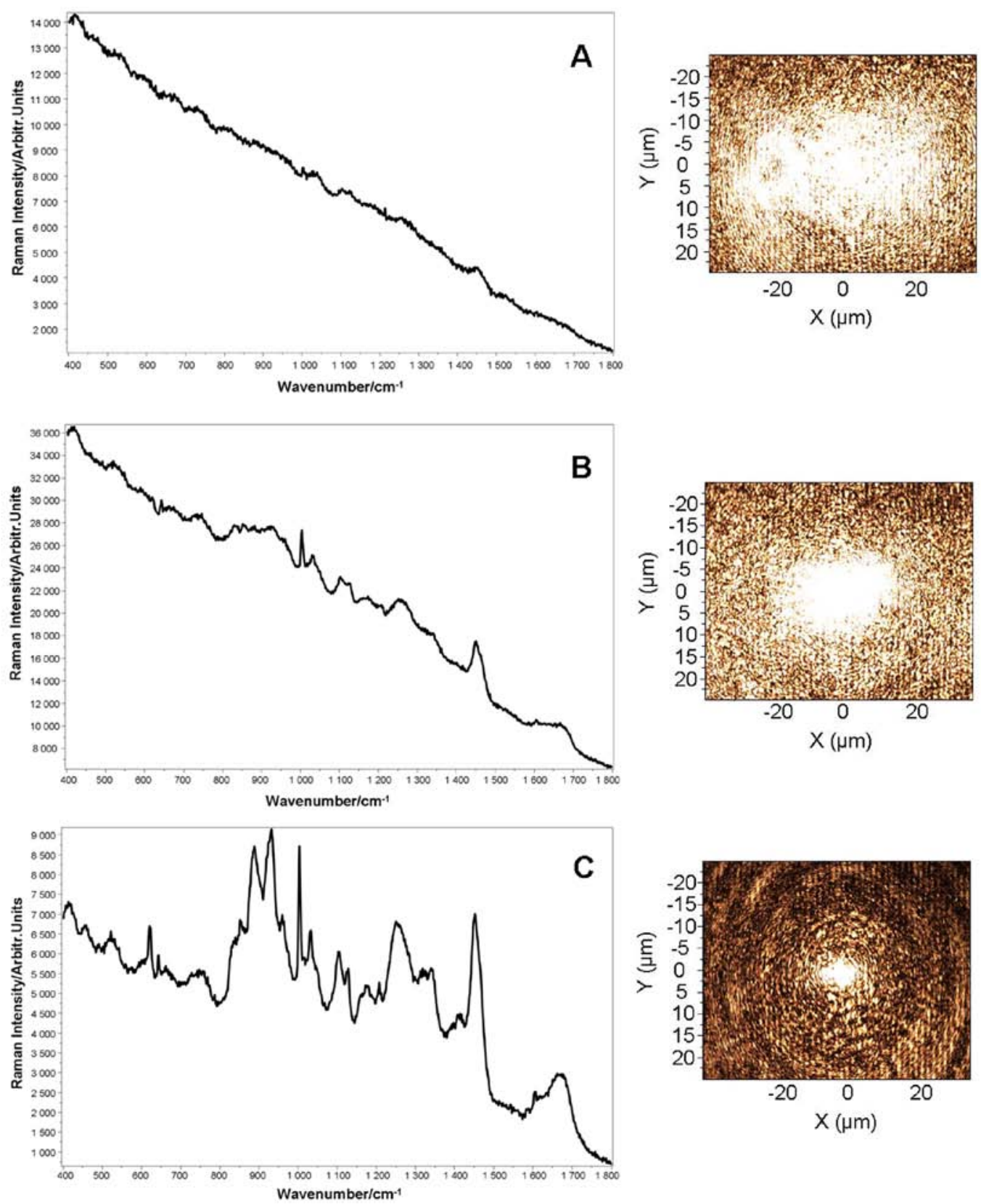

Fig 2: Raman spectra recorded from pure Kappa elastin in 3 different forms. A: Raw powder, B: disc produced by compaction and C: drop on CaF2 after solubilisation in Acetic Acid. For each Spectrum the corresponding laser spot for the focus position is displayed. 


\section{Study of immersed tissue sections}

A similar phenomenon is observable in tissue sections. When the laser spot appears ill defined on the live video camera, the recorded spectra are significantly distorted and contain a substantial background (fig.1A). Whereas with pure chemical compounds it is possible to suppress this effect by improved sample preparation techniques, this is not feasible in tissue samples, where elucidation of the spatial distribution of the chemical inhomogeneity is the objective of the study.

The tissue sections embedded in wax have to be dewaxed and rehydrated in water before analysis (see protocols in material and methods), but the samples are often kept in desiccators before analysis, with the result that they are in a dry state during measurement. As the body is made up of over $70 \%$ of water and all the tissues are highly hydrated in vivo, it is reasonable to expect that keeping the sections immersed in water during the measurements should not influence the organisation of the tissue. An immersion objective was used for the recording of the Raman spectra. The first observation made was the change in the quality of the laser spot shape at the focal point visualised on a skin section dry and after immersion (fig.3 A and B respectively). The same observation was made on the aortic samples (Data not shown).
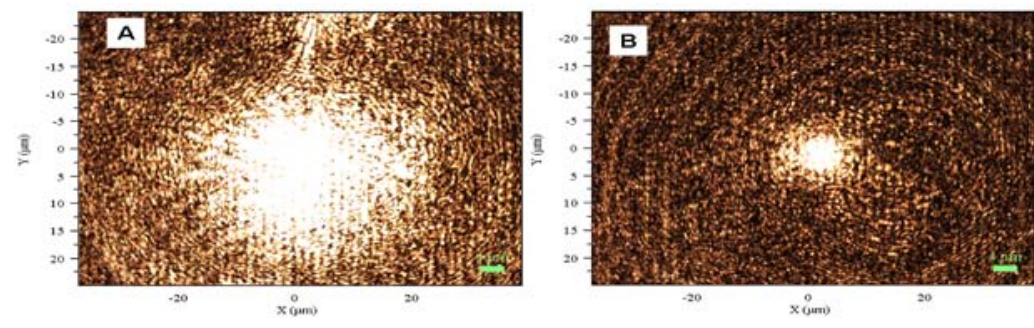

Fig.3: Laser spots obtained at the focus position on a skin sample after chemical dewaxing in the dry state (A) and immersion (B). 
The spectra recorded on the sections dry and immersed are also strongly influenced. Figure $4 \mathrm{~A}$ and $\mathrm{B}$ are two examples of recorded spectra on the skin sections from the dermis layer in the dry state. Spectrum A is dominated by the background and no Raman features can be reliably identified. Although spectrum B clearly exhibits spectral features from the tissue, a significant background is present and the peaks are not well defined. It should be noted that similar effects can be observed as a result of photothermal or photochemical damage, although usually at visible source wavelengths ${ }^{[39]}$. In this case, $785 \mathrm{~nm}$ was used as source and no change to the sample was observable by white light before or after illumination. The spectrum is a typical example of spectra that are unusable for any study of the molecular composition of the skin. The background is so strong that it is difficult to reliably identify any peaks present in the spectra. Spectra A and B highlight the problem of such interferences in the recorded spectra but the different extent of the background contributions demonstrates also the inconsistency in the effect. Therefore to develop and standardise a method for correction of the data appears quite difficult. Spectra C and D were obtained on the same sections and the same layer of the skin but immersed in distilled water. The background contribution is greatly reduced, increasing the definition of the peaks and the signal to noise ratio. It is particularly noticeable in the spectral range $800-1000 \mathrm{~cm}^{-1}, 1150-1350 \mathrm{~cm}^{-1}, 1425-1500 \mathrm{~cm}^{-1}$ and the amide I band between 1550 and $1750 \mathrm{~cm}^{-1}$. It should be noted that the spectra displayed in figure 4 are extracted from larger maps of $80 \times 50 \mu \mathrm{m}^{2}$ in all cases and are representative of the respective sample over these areas. 


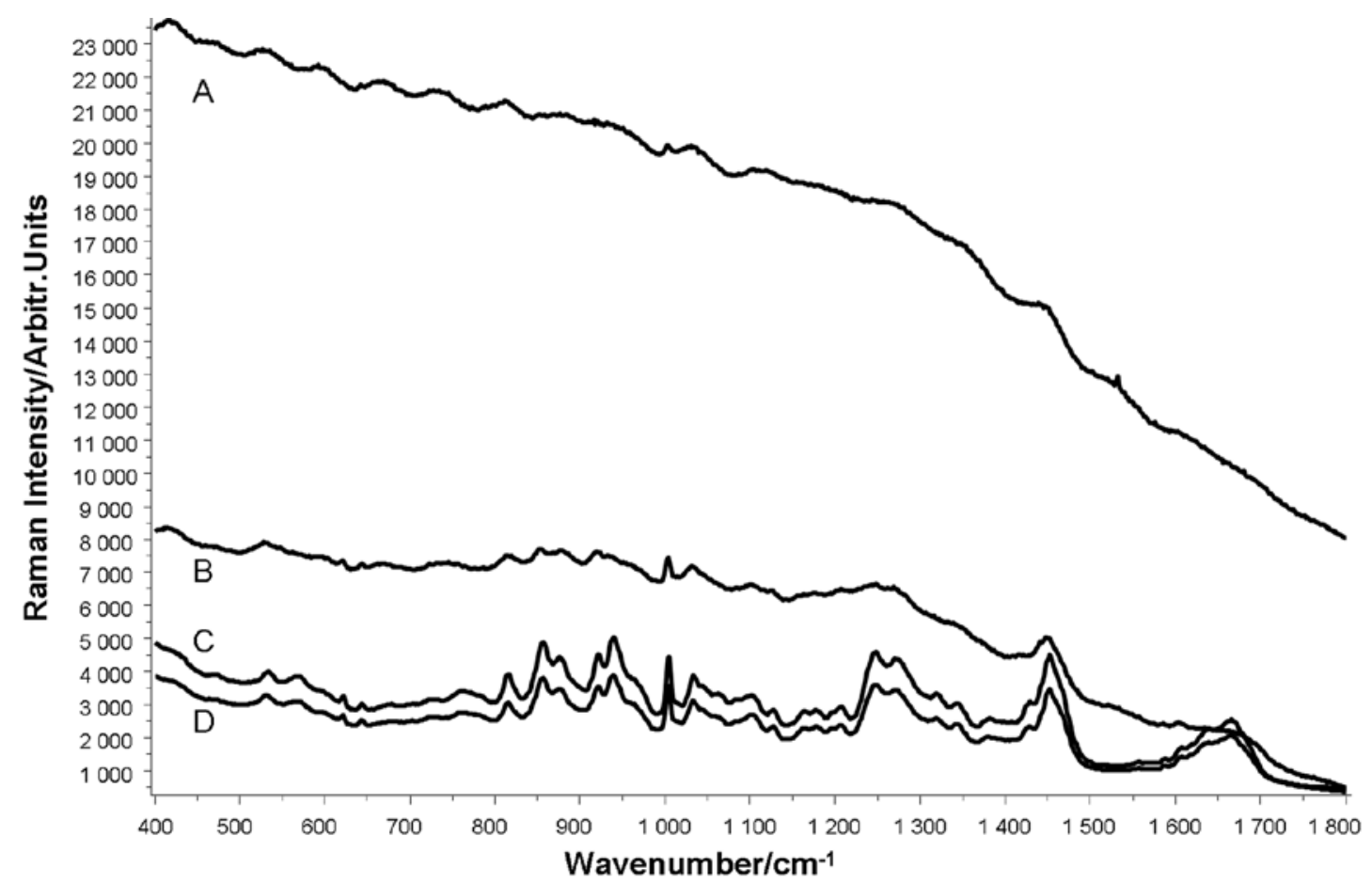

Fig 4: Example of mean recorded spectra on two different human skin samples from the thigh dry ( $A$ and $B$ ) and using the immersion objective (respectively $C$ and $D$ ). The first sample has been dewaxed in hexane ( $A$ and $D$ ), the second using xylene ( $B$ and C).

Similar observations were made in the aortic sections (fig 5). Although the recorded spectra in the dry state are less contaminated by the background, after immersion the quality of the spectra is considerably improved. The background present is significantly reduced and the definition of the peaks and signal to noise for example in the spectral windows $800-1000 \mathrm{~cm}^{-1}, 1200-1400 \mathrm{~cm}^{-1}$ and the amide I band is greatly improved. 


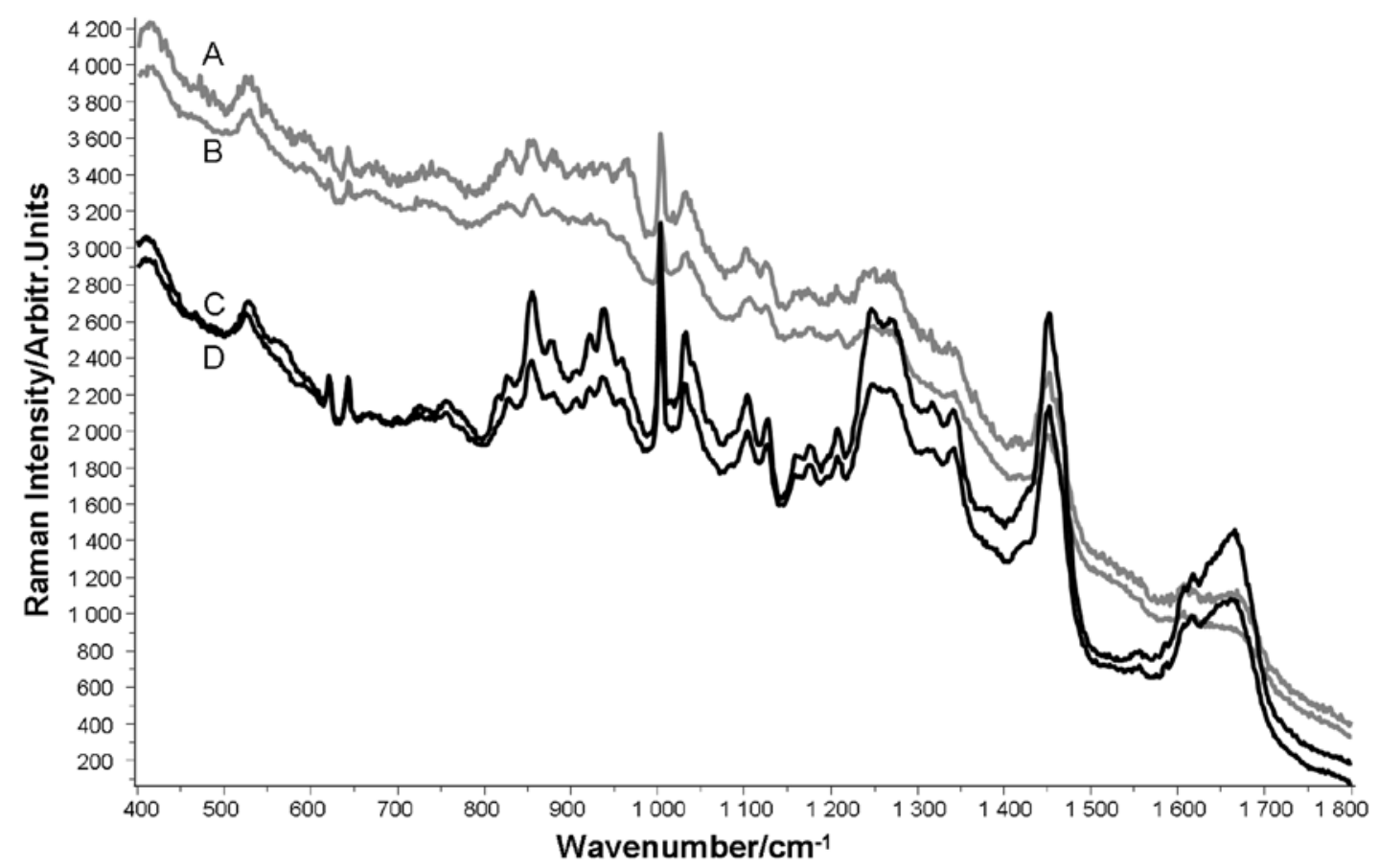

Fig 5: Example of mean recorded spectra on two different human thoracic aortas; dry (A and $B$ ) and using the immersion objective (respectively $C$ and $D$ ). The first sample has been dewaxed in hexane ( $A$ and $C$ ), the second using xylene ( $B$ and $D)$.

Similar to the studies of the Kappa-elastin samples, no chemical alteration of the samples has been affected. Notably, when dried, the previously immersed tissue sections again give rise to a large scattering background to the Raman spectra. The observed changes to the recorded signals cannot be attributed to a reduced contribution of wax within the samples as paraffin has a well defined spectrum (fig 6 A) and on its own does not contribute any significant spectral background. Neither the dry $\mathrm{CaF}_{2}$ substrate (fig. 6B), the $\mathrm{CaF}_{2}$ immersed in water, or the water alone (fig. 6C) exhibit profiles that could explain the background observed in the recorded spectra. The hydration of the tissue section obviously affects the recorded spectra and results in a reduction or elimination of the broad background. A relation clearly exists 
between the quality of the laser spot at the focal position and the presence of this abnormal deviation of the baseline.

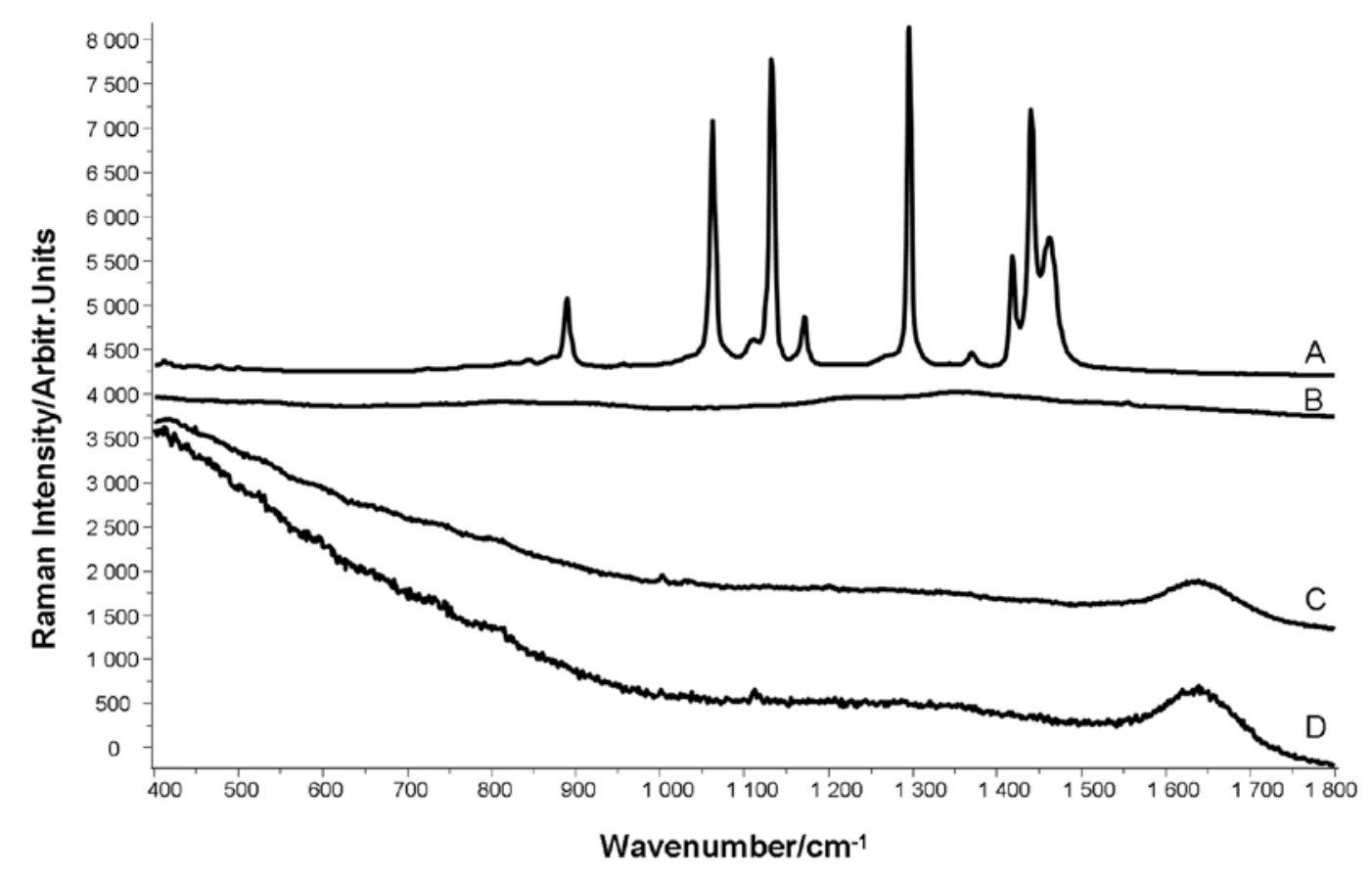

Fig. 6: A: Mean spectrum of the pure paraffin (spectra reduced 6 times to match the other spectra intensities), $B$ : Mean spectrum of the $\mathrm{CaF}_{2}$ substrate, $C$ : Mean spectrum from the CaF $\mathrm{F}_{2}$ substrate recorded using the immersion objective and D: Spectrum of the water

The background observed in Raman spectra from biological samples is commonly ascribed to intrinsic (auto) fluorescence ${ }^{[19,38]}$. However, in order to exhibit fluorescence, the sample must absorb at the excitation wavelength used. This is clearly not the case for kappa-elastin at 785nm and furthermore typical fluorescence of proteins occurs at wavelengths below $500 \mathrm{~nm}^{[40]}$. The reduction of the background observed and increase of signal to noise ratio in the recorded spectra from samples 
immersed in water clearly demonstrates that the phenomenon is not fluorescence related.

The optical properties of tissues and especially the skin are well discussed in the literature ${ }^{[41,42]}$ and can vary according to the state of the hydration of the tissues and are affected for example by aging ${ }^{[43]}$. In order to improve the quality of images recording by optical coherence tomography, optical clearing fluids are employed, which effectively match the index of refraction of the tissue with that of the voids, reducing scattering ${ }^{[44]}$. Typically for confocal fluorescence microscopy, samples are mounted between a coverslip and a glass slide, and maintained in a mounting medium, such as an aqueous buffer or a more viscous solution based on glycerol. In order to reduce as much as possible the difference between the refractive index of the different interfaces, oil immersion objectives are commonly used, reducing the refraction of the laser between the different interfaces ${ }^{[45]}$.

In Raman microscopy, the quality of the image is similarly affected by scattering and therefore morphology of the sample, and as shown in Figure 2 A, B and C. In the soluble proteins, the quality of the image is improved by producing a more homogeneous and compact surface. In the tissue samples, the images are similarly improved, in this case by immersion in distilled water. The tissue voids are filled with the higher refractive index water $(n=1.33)$ compared to air $(n=1)$. The improvement in the index matching with that of the tissue (estimated at 1.4 for the dermis layer ${ }^{[46-48]}$ ) significantly reduces the scatter, by changing the effective optical morphology and improves the image quality.

The spectral background is similarly correlated with the sample morphology, or effective morphology. This morphological dependence of the Raman background 
points towards diffuse or Mie scattering as its origin. The white aspect of the powder and that of the tissue sections is a clear indicator of a morphology in which the feature dimensions are of the order of or greater than the incident wavelength, giving rise to such scattering. Mie scattering is however elastic and the question remains as to why such scattered radiation appears spectrally dispersed in the Raman spectrum. In the backscattering geometry employed by microscopic Raman systems, efficient spectral harvesting relies on the collimation of the backscattered radiation from the samples. Diffusely scattered radiation is not collimated and, impinging on the holographic notch filter, whose transmittance spectrum is strongly angle dependent, can be admitted to the spectrometer as stray light. This stray light is distributed across the CCD array in an apparently spectrally dispersed fashion. It is unlikely however that stray light from the source laser is the sole contributor to the background, and that lack of collimation of the Raman bands themselves broadens them, reducing the peak intensity and contributing to the background. Reduction of the Mie scattering in immersion results in a substantially reduced background and increased signal intensity and therefore increased signal to noise ratio.

\section{Data pre-processing facilitated}

Examples of mean spectra from the aorta and skin are displayed in figure $7 \mathrm{~A}$ and $\mathrm{B}$ respectively. Commonly, the first correction to be applied is the subtraction of the substrate signal from the recorded spectra. As the signal for the $\mathrm{CaF}_{2}$ is weak (fig.6 B), the spectra corresponding to the substrate is mostly influenced by the signal of the water (fig.6 C and D), replotted in figure 7 for ease of comparison relative intensities. The second correction to be applied is related to the instrument response. The signal 
needed for the correction is calculated after recording the signal from the SMR specific for the $785 \mathrm{~nm}$ laser (fig.7 C) (see materials and methods for more details). The spectra obtained after correction are presented in figure 8. Spectra 8A and B are those recorded from the immersed samples whereas spectra 8C and D are from the dry tissue. At this stage no baseline correction has been applied on the spectra but it is noticeable that spectra A and B have negligible baseline contributions. A slight off set is present between the spectra from the skin and aorta probably due to different density of the tissue. Concerning the recorded spectra from dry sections, the baseline is still strongly distorted. These spectra are difficult to correct and the ratio between the different peaks is inconsistent. Considering the shape of the spectra, a linear baseline correction is not appropriate, and although higher order polynomial functions can be applied, their accuracy and therefore impact on the final spectral shape is not known. This clearly highlights the advantage of using the immersion approach for tissue section analysis after dewaxing. 


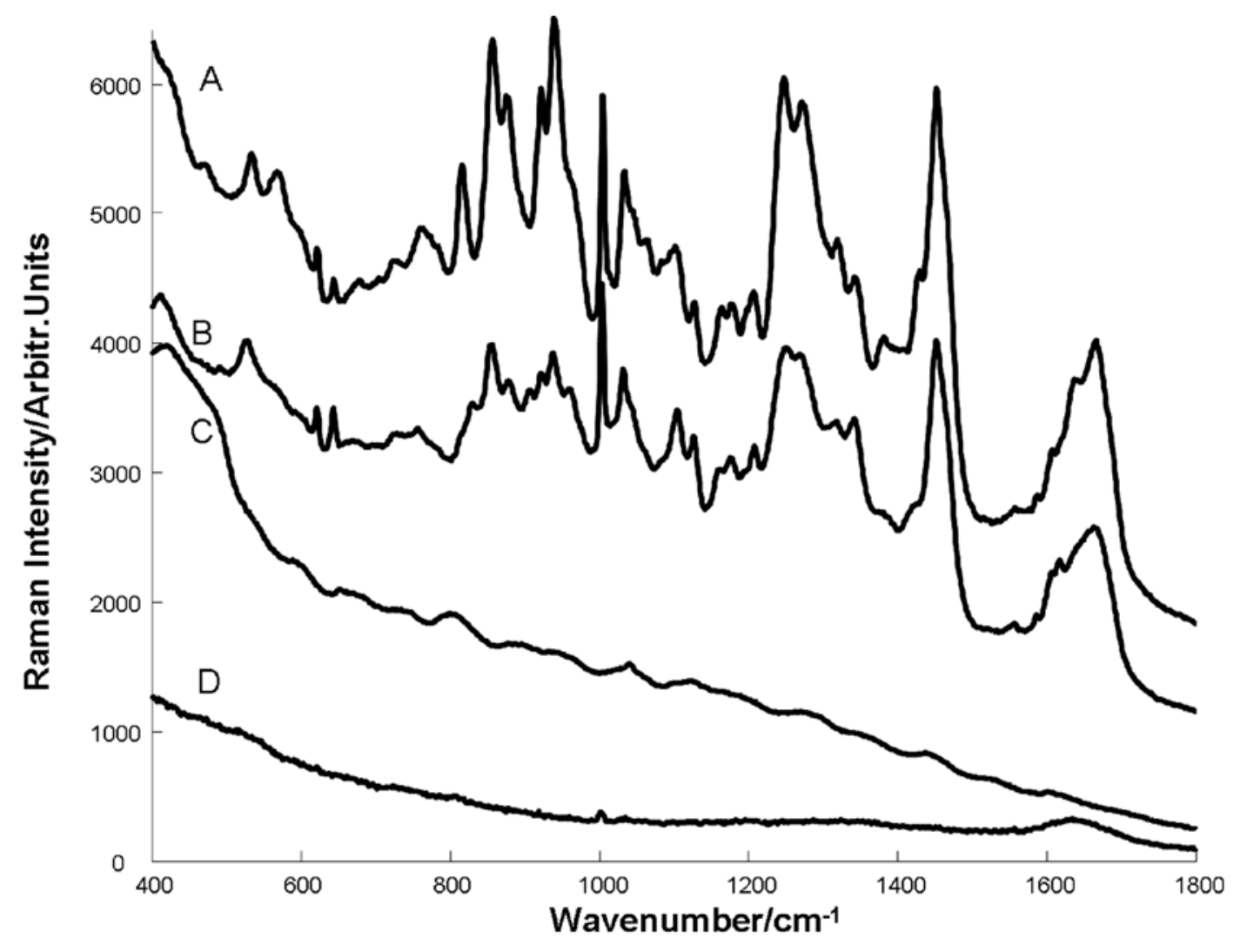

Fig.7: Pre-processing of the data. A: Spectrum from human skin from the thigh after dewaxing in xylene, B: Spectrum from human thoracic aorta after dewaxing using hexane, C: Signal use to correct the instrument response (spectra reduced 15 times to match the other spectra intensities). D: Signal of the background ( $\mathrm{CaF}_{2}$ recorded using the immersion objective). 

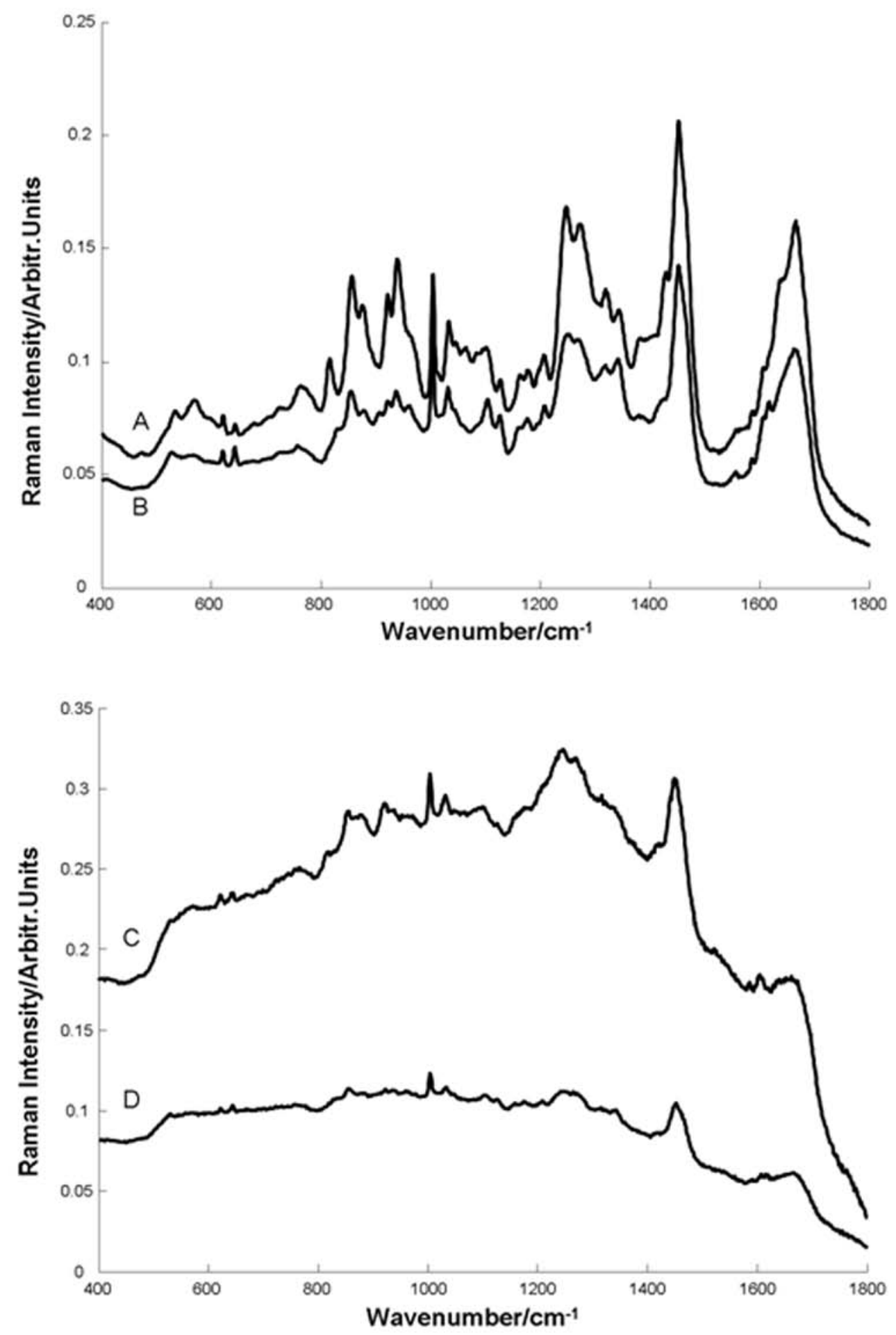

Fig. 8: Data after pre-processing. Step one: subtraction of the background, Step 2: division by the standard signal to correct instrument response. A: Spectrum from immersed human skin from the thigh after dewaxing in xylene, B: Spectrum from immersed human thoracic aorta after dewaxing using hexane, C: Spectrum from dry human skin from the thigh after dewaxing in xylene D: Spectrum from dry human thoracic aorta after dewaxing using hexane. 


\section{Conclusion}

Using chemically pure proteins as model systems, it is clearly demonstrated that the broad background observed in Raman microspectroscopy can be dependent on the sample morphology. Although a fluorescing material will significantly contribute to the background, materials non resonant with the excitation source can contribute by stray Mie scattered light from the source and the Raman lines themselves, which is dispersed across the CCD. In tissues, immersion in water provides a degree of index matching, improving the effective morphology and reducing the scattering and therefore the Raman background. In dewaxed samples, the background is reduced to minimal levels, greatly facilitating preprocessing. Eliminating the background at the measurement stage greatly enhances the reliability of the preprocessing, reducing it to removal of measurable contributions, help to increase the accuracy of and confidence in the data sets recorded.

\section{Acknowledgements}

This research was supported by the National Biophotonics and Imaging Platform (NBIP) Ireland funded under the Higher Education Authority PRTLI (Programme for Research in Third Level Institutions) Cycle 4, co-funded by the Irish Government and the European Union. 


\section{REFERENCES}

[1] A. Beljebbar, O. Bouche, M. D. Diebold, P. J. Guillou, J. P. Palot, D. Eudes, M. Manfait, Crit Rev Oncol Hematol 2009; 72, 255.

[2] J. L. Bruggink, R. Meerwaldt, G. M. van Dam, J. D. Lefrandt, R. H. Slart, R. A. Tio, A. J. Smit, C. J. Zeebregts, Int J Cardiovasc Imaging; 26, 111.

[3] M. E. Darvin, S. F. Haag, M. C. Meinke, W. Sterry, J. Lademann, J Biophotonics.

[4] A. Downes, R. Mouras, A. Elfick, J Biomed Biotechnol; 2010, 101864.

[5] F. Draux, P. Jeannesson, A. Beljebbar, A. Tfayli, N. Fourre, M. Manfait, J. Sule-Suso, G. D. Sockalingum, Analyst 2009; 134, 542.

[6] G. J. Puppels, F. F. de Mul, C. Otto, J. Greve, M. Robert-Nicoud, D. J. ArndtJovin, T. M. Jovin, Nature 1990; 347, 301.

[7] S. Venyaminov, F. G. Prendergast, Anal Biochem 1997; 248, 234.

[8] K. Varmuza, Introduction to multivariate statistical analysis in chemometrics, CRC Press, New york, 2009.

[9] F. Bonnier, D. Bertrand, S. Rubin, L. Venteo, M. Pluot, B. Baehrel, M. Manfait, G. D. Sockalingum, Analyst 2008; 133, 784.

[10] E. Ly, O. Piot, A. Durlach, P. Bernard, M. Manfait, Analyst 2009; 134, 1208.

[11] E. Ly, O. Piot, R. Wolthuis, A. Durlach, P. Bernard, M. Manfait, Analyst 2008; 133, 197.

[12] Y. Oshima, H. Shinzawa, T. Takenaka, C. Furihata, H. Sato, J Biomed Opt; 15, 017009.

[13] R. Wolthuis, A. Travo, C. Nicolet, A. Neuville, M. P. Gaub, D. Guenot, E. Ly, M. Manfait, P. Jeannesson, O. Piot, Anal Chem 2008; 80, 8461.

[14] B. Mohlenhoff, M. Romeo, M. Diem, B. R. Wood, Biophys J 2005; 88, 3635.

[15] P. Bassan, H. J. Byrne, J. Lee, F. Bonnier, C. Clarke, P. Dumas, E. Gazi, M. D. Brown, N. W. Clarke, P. Gardner, Analyst 2009; 134, 1171.

[16] M. J. Romeo, M. Diem, Vib Spectrosc 2005; 38, 115.

[17] S. N. Thennadil, H. Martens, A. Kohler, Appl Spectrosc 2006; 60, 315.

[18] P. Bassan, A. Kohler, H. Martens, J. Lee, H. J. Byrne, P. Dumas, E. Gazi, M. Brown, N. Clarke, P. Gardner, Analyst; 135, 268.

[19] C. A. Lieber, A. Mahadevan-Jansen, Appl Spectrosc 2003; 57, 1363.

[20] E. B. Hanlon, R. Manoharan, T. W. Koo, K. E. Shafer, J. T. Motz, M. Fitzmaurice, J. R. Kramer, I. Itzkan, R. R. Dasari, M. S. Feld, Phys Med Biol 2000; 45, R1.

[21] B. D. Beier, A. J. Berger, Analyst 2009; 134, 1198.

[22] P. J. Caspers, G. W. Lucassen, G. J. Puppels, Biophys J 2003; 85, 572.

[23] N. D. Magee, J. S. Villaumie, E. T. Marple, M. Ennis, J. S. Elborn, J. J. McGarvey, J Phys Chem B 2009; 113, 8137.

[24] J. T. Motz, S. J. Gandhi, O. R. Scepanovic, A. S. Haka, J. R. Kramer, R. R. Dasari, M. S. Feld, J Biomed Opt 2005; 10, 031113.

[25] E. Ó. Faoláin, Dublin Institute of Technology (Dublin), 2006.

[26] E. M. Kanter, E. Vargis, S. Majumder, M. D. Keller, E. Woeste, G. G. Rao, A. Mahadevan-Jansen, J Biophotonics 2009; 2, 81.

[27] J. D. Bancroft, M. Gamble, Theory and Practice of Histological Techniques, Churchill Livingstone, London, 2002.

[28] R. Drury, E. Wallington, Carleton's Histological technique, Oxford University Press, Oxford, 1980. 
[29] E. O. Faolain, M. B. Hunter, J. M. Byrne, P. Kelehan, H. A. Lambkin, H. J. Byrne, F. M. Lyng, J Histochem Cytochem 2005; 53, 121.

[30] A. Tfayli, C. Gobinet, V. Vrabie, R. Huez, M. Manfait, O. Piot, Appl Spectrosc 2009; 63, 564.

[31] G. J. Puppels, W. Colier, J. H. F. Olminkhof, C. Otto, F. F. M. d. Mul, J. Greve, Journal of Raman Spectroscopy 1991; 22, 217.

[32] M. Jacob, W. Hornebeck, Front. Matrix Biol. 1985, 92.

[33] S. J. Choquette, E. S. Etz, W. S. Hurst, D. H. Blackburn, S. D. Leigh, Appl Spectrosc 2007; 61, 117.

[34] J. M. Clark, S. Glagov, Arteriosclerosis 1985; 5, 19.

[35] G. F. Odland, in In Physiology, Biochemistry and molecular Biology of the Skin, (Eds: L. A. Goldsmith), Oxford University Press, New York, 1991, pp. 362.

[36] L. Debelle, A. J. Alix, M. P. Jacob, J. P. Huvenne, M. Berjot, B. Sombret, P. Legrand, J Biol Chem 1995; 270, 26099.

[37] L. Debelle, A. J. Alix, S. M. Wei, M. P. Jacob, J. P. Huvenne, M. Berjot, P. Legrand, Eur J Biochem 1998; 258, 533.

[38] J. Zhao, H. Lui, D. I. McLean, H. Zeng, Appl Spectrosc 2007; 61, 1225.

[39] G. J. Puppels, J. H. Olminkhof, G. M. Segers-Nolten, C. Otto, F. F. de Mul, J. Greve, Exp Cell Res 1991; 195, 361.

[40] R. Redmond, Handbook of Biomedical Fluorescence "Introduction to Fluorescence and Photophysics", Marcel Dekker, NewYork, 2003.

[41] H. Ding, J. Q. Lu, W. A. Wooden, P. J. Kragel, X. H. Hu, Phys Med Biol 2006; 51, 1479.

[42] M. G. Ghosn, S. H. Syed, N. A. Befrui, M. Leba, A. Vijayananda, N. Sudheendran, K. V. Larin, Laser Physics 2009; 19, 1272.

[43] M. A. Calin, S. V. Parasca, Lasers Med Sci; 25, 269.

[44] M. Brezinski, K. Saunders, C. Jesser, X. Li, J. Fujimoto, Circulation 2001; 103, 1999.

[45] A. Egner, S. W. Hell, Handbook of Biological Confocal Microscopy, SpringerScience / Business Media, New York, 2006.

[46] A. Knuttel, M. Boehlau-Godau, J Biomed Opt 2000; 5, 83.

[47] M. Rajadhyaksha, S. Gonzalez, J. M. Zavislan, R. R. Anderson, R. H. Webb, J Invest Dermatol 1999; 113, 293.

[48] G. J. Tearney, M. E. Brezinski, J. F. Southern, B. E. Bouma, M. R. Hee, J. G. Fujimoto, Opt Lett 1995; 20, 2258. 